\title{
Extending the census at the bottom of the stellar mass function in Chamaeleon I*
}

\author{
F. Comerón ${ }^{1}$, B. Reipurth ${ }^{2}$, A. Henry ${ }^{3}$, and M. Fernández ${ }^{4}$ \\ ${ }^{1}$ European Southern Observatory, Karl-Schwarzschild-Strasse 2, 85748 Garching, Germany \\ 2 Institute for Astronomy, University of Hawaii, 2680 Woodlawn Drive, Honolulu, HI 96822, USA \\ e-mail: reipurth@ifa.hawaii.edu \\ 3 Department of Physics \& Astronomy, UCLA, Los Angeles, CA 90095-1547, USA \\ e-mail: alaina@astro.ucla.edu \\ 4 Instituto de Astrofísica de Andalucía, CSIC, Apdo. 3004, 18080 Granada, Spain \\ e-mail: matilde@iaa.es
}

Received 8 September 2003 / Accepted 15 December 2003

\begin{abstract}
We present the results of a deep, wide field objective prism survey of the entire Chameleon I cloud, followed by long slit spectroscopy of objects with detected $\mathrm{H} \alpha$ emission that were either previously unidentified, or suspected to be members only on the basis of their mid-infrared emission. We identify 9 new members and confirm 9 objects already suspected as members, with spectral types ranging from late $\mathrm{K}$ to M8.5. The latter limit corresponds to an object with an estimated mass of $0.03 M_{\odot}$, making it the latest-type brown dwarf spectroscopically confirmed so far in Chamaeleon I. A comparison with theoretical premain sequence tracks indicates an age of most of the members between 1 and $5 \mathrm{Myr}$, consistent with previous studies. However, we find that the objects with $\mathrm{H} \alpha$ equivalent widths exceeding $100 \AA$ tend to have apparent ages above $5 \mathrm{Myr}$, and in the two most extreme cases their positions in the temperature-luminosity diagram, if interpreted at face value, would place them below the main sequence. These two extreme objects display surprising differences in their emission line spectra in spite of the otherwise very similar broad-band spectral energy distributions and spectral types, being strongly dominated by accretion-tracing and outflow-tracing emission lines, respectively. We interpret the identification of an apparently underluminous object with strong accretion signatures and only weak outflow signatures as a further support, already discussed in previous works, for the apparent underluminosity of objects with very large $\mathrm{H} \alpha$ equivalent widths being a real, intrinsic feature rather than being due to partial blocking by an edge-on disk. Given that $\mathrm{H} \alpha$ emission is the most common feature among young stellar objects we consider that the present work is an important contribution towards a complete census of the Chamaeleon I star forming region down to the hydrogen-burning limit.
\end{abstract}

Key words. stars: pre-main sequence - stars: low-mass, brown dwarfs - stars: winds, outflows

\section{Introduction}

Dedicated wide-field observations and all-sky surveys sensitive enough to reveal young brown dwarfs in nearby star forming regions have opened up the possibility of addressing a variety of questions related to the collective properties of these objects and their early evolution. Issues such as the period over which a cloud forms stars, the existence and efficiency of triggering mechanisms, spatial variations in the mass function, the processes responsible for the presence of young stars in zones nearly devoid of molecular gas, the incidence and statistics of multiplicity, or the dependency of the star formation process on environments of different densities, may be studied only by

Send offprint requests to: F. Comerón,

e-mail: fcomeron@eso.org

* Based on observations obtained at the European Southern Observatory using the ESO New Technology Telescope on La Silla (programme 71.C-0432(A)). means of complete samples covering the whole range of conditions encountered in a star forming region, not only those of its most active star forming cores. In addition, the study of individual young, very low mass objects has revealed a remarkable diversity among them, possibly related to their accretion and mass loss activities and their possible impact on intrinsic properties and evolutionary paths. Understanding their early evolution benefits from the identification of cases in which certain signatures are more readily visible than in others, allowing the isolation of the contributions of different phenomena that determine the appearance of the object. Enlarging the available samples is needed to probe such diversity.

Moderately deep imaging surveys of nearby star forming regions are very efficient in selecting brown dwarf candidates (e.g. López Martí 2003). Nevertheless, visible or nearinfrared spectral classification remains as the most reliable way to robustly determine intrinsic properties. For lightly obscured 
objects, the visible spectral region offers a wealth of information on the photospheric emission and displays telltale signatures of mass accretion and mass loss common among young stellar objects (e.g. Cabrit et al. 1990; Hamann et al. 1994; Hartigan et al. 1995, and references in those papers), down to the star/brown dwarf boundary (Fernández \& Comerón 2001; Comerón et al. 2003). Near-infrared classification is a useful alternative for objects with higher extinctions (see e.g. Greene \& Meyer 1995; Greene \& Lada 1996; Luhman \& Rieke 1998; Carpenter et al. 1997; Natta et al. 2002 and the recent work of Gómez \& Persi 2002 and Gómez \& Mardones 2003 on Chamaeleon I), yielding accuracies that approach those attainable at visible wavelengths when high signal-to-noise ratios are available (Gorlova et al. 2003).

The Chamaeleon I complex has been a frequent target of the searches for young stellar objects with low masses. Wide area surveys have been performed in X-rays in combination with optical spectroscopy (Huenemoerder et al. 1994; Lawson et al. 1996; Alcalá et al. 1995, 1997; Covino et al. 1997), in the thermal infrared searching for objects surrounded by warm dust (Baud et al. 1984; Prusti et al. 1991; Persi et al. 2000 ), and in the near infrared searching for objects with $K$-band excess arising from the innermost regions of circumstellar disks (Cambrésy et al. 1998; Oasa et al. 1999; Persi et al. 1999; Gómez \& Kenyon 2001) or for near-infrared variability (Carpenter et al. 2002). Deeper surveys of the areas with a high density of objects, able to penetrate well into the substellar domain, have been carried out using similar identification techniques (Neuhäuser \& Comerón 1998; Oasa et al. 1999; Comerón et al. 1999, 2000). Follow-up imaging observations of the best brown dwarf candidates have searched for even lower mass companions (Neuhäuser et al. 2002) or rotation possibly induced by variability (Joergens et al. 2003), while spectroscopy in the visible (Neuhäuser \& Comerón 1999; Joergens \& Guenther 2001) and the near- and mid-infrared (Gürtler et al. 1999; Natta \& Testi 2001; Apai et al. 2002; Gómez \& Mardones 2003) have provided detailed information on individual objects detected in previous surveys.

A fruitful approach intermediate between imaging surveys and their spectroscopic follow-up is offered by slitless spectroscopy around the $\mathrm{H} \alpha$ region. In lightly obscured populations such as most of the Chamaeleon star forming complex (Schwartz 1991), $\mathrm{H} \alpha$ objective prism surveys have been a traditional technique for member identification (e.g. Henize \& Mendoza 1973; Schwartz 1977; Hartigan 1993). It remains as a very competitive one too: objective prism spectra provide a rough estimate of the spectral type, thus greatly reducing the contamination by unrelated sources that affects other techniques in ways that are difficult to quantify, while $\mathrm{H} \alpha$ yields an indicator most usually related to accretion activity. Moreover, $\mathrm{H} \alpha$ surveys produce highly complete samples of young stellar objects even at very low masses (Comerón et al. 1999), thus providing one of the most effective ways to probe the entire initial mass function in star forming regions.

In this paper we report on the results of a deep objective prism survey of the entire Chamaeleon I cloud, followed by long-slit spectroscopy of the candidate members detected, with the goal of extending the census of spectroscopically confirmed
Table 1. List of Schmidt films.

\begin{tabular}{rrr}
\hline \hline Date & No. & Exp. time \\
\hline 15 Feb. 1996 & 12178 & $150 \mathrm{~min}$ \\
13 April 1996 & 12312 & $150 \mathrm{~min}$ \\
14 April 1996 & 12316 & $10 \mathrm{~min}$ \\
16 Dec. 1996 & 12903 & $45 \mathrm{~min}$ \\
13 Jan. 1996 & 12955 & $90 \mathrm{~min}$ \\
15 Jan. 1997 & 12961 & $90 \mathrm{~min}$ \\
6 Feb. 1997 & 12987 & $90 \mathrm{~min}$ \\
\hline
\end{tabular}

members of the star forming region towards very low masses and luminosities. We discuss the properties of new members identified in this way and complement them wherever possible with results available at other wavelengths. Special attention is given to a reduced number of objects in our sample with strong emission lines relative to a faint continuum. Our observations are described in Sect. 2, and the results and their implications are presented in Sect. 3. The main results of this work are summarized in Sect. 4.

\section{Observations}

\subsection{Objective prism spectroscopy and new member identification}

The selection of candidate members was performed on a deep survey obtained at the ESO $1 \mathrm{~m}$ Schmidt telescope between February 1996 and February 1997. The telescope was equipped with an objective prism of $4^{\circ}$ opening angle, yielding a reciprocal dispersion at $\mathrm{H} \gamma$ of $450 \AA \mathrm{mm}^{-1}$, or $800 \AA \mathrm{mm}^{-1}$ at $\mathrm{H} \alpha$ (Reipurth 1996). The films were based on the very sensitive fine-grained Kodak Tech-Pan 4415 emulsion, offering a significant advantage compared to the old IIIaF glass plates. We used a series of exposures of 10, 45, 90 and $150 \mathrm{~min}$, with no widening, thus permitting a complete study of $\mathrm{H} \alpha$ emission from the brightest members to extremely faint objects. Table 1 lists information about the films employed in this survey. Combined with the unique 25 square degree field of the ESO Schmidt telescope, our survey is thus the largest and deepest so far in Chamaeleon I. During this survey, we identified nearly all the already known members in the region listed by Carpenter et al. (2002), including three of the brown dwarfs found by Comerón et al. (2000), thus demonstrating that our technique probes well into the substellar regime in the Chamaeleon I cloud. We also identified 24 new objects with either obvious or suspected $\mathrm{H} \alpha$ emission, which we denote $\mathrm{ESO}-\mathrm{H} \alpha 552$ to 575 , in continuation of our previous surveys of $\mathrm{H} \alpha$ emission line stars in southern star forming regions (Pettersson \& Reipurth 1994; Reipurth et al. 2003). Finding charts from red CCD images are shown in Fig. 1. The identification of the $\mathrm{H} \alpha$ emission candidates on the films was done visually with a binocular microscope, and their coordinates were measured on red Digitized Sky Survey images. The traces of individual spectra were approximately $30^{\prime \prime}$ long aligned in the North-South direction, and none of them suffers from overlap with the spectrum of any nearby object located in that same direction. 


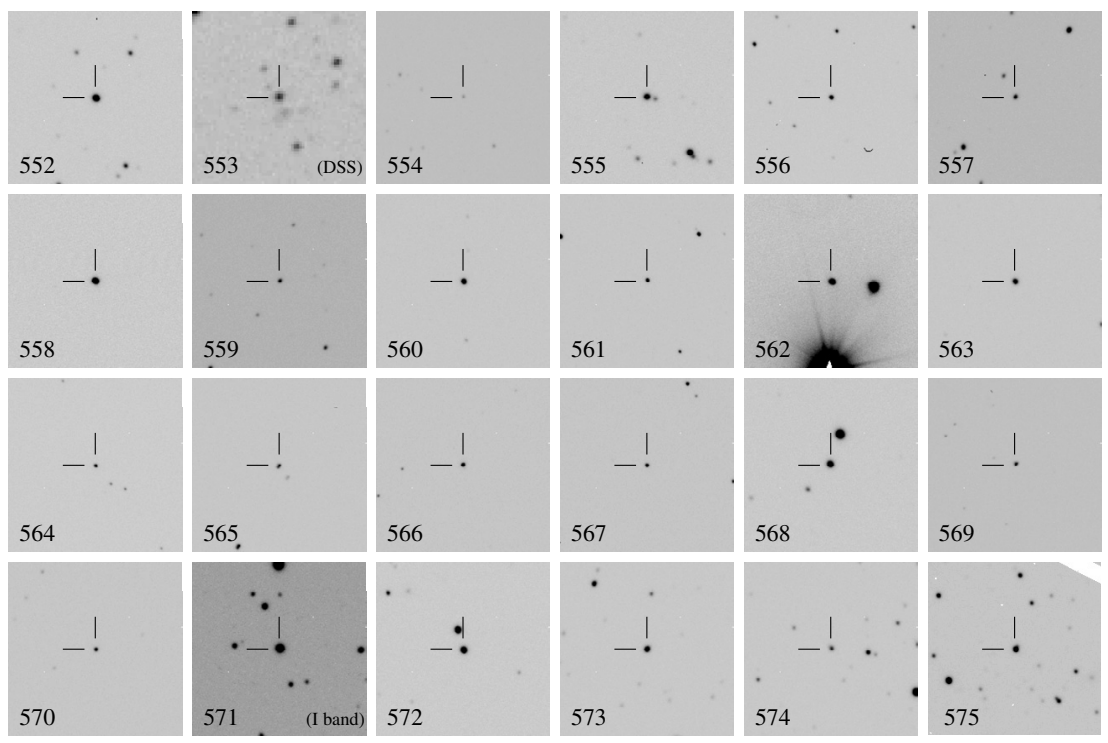

Fig. 1. Finding charts for the $24 \mathrm{H} \alpha$ emission candidates ESO-H $\alpha 552$ to 575 . The charts are based on acquisition images through a $R$-band filter, except for ESO-H $\alpha$ 571, where an I-band image is shown. One star, ESO-H $\alpha$ 553, was not observed, and a red DSS image is shown. All fields shown are 66 arcsec on a side, and North is up and East is left.

\subsection{Long-slit spectroscopy and VRI imaging}

Our objective prism observations were designed to provide identifications of objects for later follow-up, rather than calibrated flux measurements. In particular, they are very poorly suited to extract quantitative information such as line equivalent widths, mainly due to the nonlinear response of the film and, for the faint objects on which we focus in the present paper, to the weak signal produced by the underlying continuum. Therefore, follow-up long-slit spectroscopy in the visible of 23 of the newly identified members was obtained on the nights of 1 and 2 April 2003 using EMMI, the visible imager and low-to-medium resolution spectrograph at the ESO New Technology Telescope (NTT). An additional possible member, ESO-H $\alpha$ 553, was not included in our spectroscopic observations and is not discussed in this paper. No previous spectroscopy in the visible exists in the literature for any of the objects discussed here.

We used a grism yielding a dispersion of $2.4 \AA$ pix $^{-1}$ over the $6000 \AA<\lambda<10000 \AA$ range, adequate for spectral classification purposes. Two spectra of each object were obtained to facilitate the removal of spurious features. The exposure time for each spectrum ranged from 30 to $900 \mathrm{~s}$, depending on the $R$ magnitude of the object as roughly estimated from the Schmidt telescope observations. The spectrophotometric standard LTT4364 (Hamuy et al. 1992) was also observed to enable relative flux calibration of our program stars. No correction for telluric absorption was attempted. Each set of two spectra was followed by imaging observations of the target field in $V, R_{\mathrm{C}}$, and $I_{\mathrm{C}}$, which were flux-calibrated through observations of the field of PG1047+003 (Landolt 1992). The typical point spread function of our observations has a full width at half maximum of $0^{\prime \prime} 8$, and all our sources appear point-like, with the exception of ESO-H $\alpha 575$ that has a barely resolved, fainter companion $1^{\prime \prime} 2$ to the northwest. The positions and magnitudes measured for each object, as well as the spectral types assigned (see Sect. 3.1) are given in Table 2.

\subsection{Near-infrared imaging}

The 2MASS All Sky Survey contains $J H K_{\mathrm{S}}$ photometry of all the objects included in this paper. Our discussion on their infrared properties is thus based on the 2MASS data. We could also obtain $J H K_{\mathrm{S}}$ imaging for a reduced number of targets at the end of our second observing night at the NTT, which can be compared to the 2MASS photometry for possible variability. Photometric data from both sources are given in Table 2. The infrared images were obtained using SOFI, the infrared camera and spectrograph at the NTT, and calibrated with observations of the standard star S064-F (Persson et al. 1998).

\section{Results}

\subsection{Spectral types and membership}

The long-slit spectroscopy confirms that all the objects have late-type spectra and in most cases also $\mathrm{H} \alpha$ emission. The spectra of 18 objects that we classify as members of Chamaeleon I are shown in Figs. 2 and 3. Membership is assigned based on the existence of $\mathrm{H} \alpha$ emission as well as on the weakness of the gravity-dependent NaI $8190 \AA$ feature, which is frequently used as a discriminator between young late-type objects in their early contraction stages or stars in young open clusters, and more evolved stars of the same spectral types closer to the main sequence (see Martín et al. 1996; Béjar et al. 1999, and references therein). The presence or absence of Li $6707 \AA$ absorption, commonly used as a test of the youth of very low mass objects (e.g. Martín et al. 1999), cannot be established at the resolution and signal-to-noise ratio of our observations.

The spectral classification has been performed as described in Comerón et al. (2003), and is based on the overall 
Table 2. Positions, photometry, and spectral types of the observed stars.

\begin{tabular}{|c|c|c|c|c|c|c|c|c|c|c|c|c|}
\hline $\begin{array}{l}\text { Object }^{1} \\
(\mathrm{ESO}-\mathrm{H} \alpha)\end{array}$ & $\alpha(2000)$ & $\delta(2000)$ & $V$ & $R_{\mathrm{C}}$ & $I_{\mathrm{C}}$ & $J$ & $\begin{array}{c}H \\
\text { (2MASS) }\end{array}$ & $\overline{K_{\mathrm{S}}}$ & $J$ & $\begin{array}{c} \\
\text { pril } 2003\end{array}$ & $K_{\mathrm{S}}$ & Sp. type \\
\hline \multicolumn{13}{|c|}{ Members with $E W(\mathrm{H} \alpha)<100 \AA$} \\
\hline 552 & $10: 55: 10.5$ & $-77: 30: 55$ & 17.49 & 15.75 & 13.98 & 11.881 & 11.112 & 10.801 & & & & M5 \\
\hline 554 & $11: 01: 32.9$ & $-77: 18: 27$ & 22.62 & 19.78 & 17.66 & 14.631 & 14.081 & 13.545 & & & & M8.5 \\
\hline 558 & $11: 04: 42.6$ & $-77: 41: 58$ & 16.88 & 15.31 & 13.68 & 11.814 & 11.002 & 10.642 & & & & M4. $5^{2}$ \\
\hline 559 & $11: 06: 26.3$ & $-76: 33: 42$ & 20.25 & 17.95 & 15.92 & 13.009 & 12.007 & 11.486 & & & & M6 \\
\hline 560 & $11: 07: 38.3$ & $-77: 47: 17$ & 18.37 & 16.44 & 14.61 & 12.240 & 11.399 & 11.027 & & & & M5 \\
\hline 561 & 11:08:00.7 & $-77: 15: 32$ & 20.09 & 17.49 & 15.51 & 12.522 & 11.646 & 11.167 & & & & M6.5 \\
\hline 564 & $11: 08: 55.7$ & $-76: 32: 42$ & 20.12 & 17.85 & 15.96 & 13.060 & 12.059 & 11.439 & & & & M6 \\
\hline 565 & 11:09:43.3 & $-77: 25: 59$ & 20.93 & 17.97 & 15.78 & 12.329 & 11.175 & 10.552 & & & & M6 \\
\hline 566 & 11:09:45.2 & $-77: 40: 34$ & 19.66 & 17.24 & 15.16 & 12.355 & 11.450 & 11.033 & & & & M6.5 \\
\hline 568 & $11: 10: 51.6$ & $-77: 18: 04$ & 18.11 & 16.21 & 14.41 & 12.044 & 11.101 & 10.748 & 12.05 & 11.08 & 10.73 & M4.5 \\
\hline 570 & $11: 12: 04.4$ & $-77: 26: 02$ & 20.08 & 17.82 & 15.91 & 13.626 & 12.587 & 11.842 & 13.12 & 12.16 & 11.50 & M6 \\
\hline 571 & $11: 14: 29.9$ & $-76: 25: 40$ & 17.73 & & 14.45 & 12.567 & 11.902 & 11.607 & 12.55 & 11.90 & 11.62 & M5.5 \\
\hline 572 & $11: 15: 22.7$ & $-77: 24: 05$ & 17.38 & 15.64 & 13.88 & 11.764 & 11.132 & 10.824 & 11.81 & 11.13 & 10.89 & M5.5 \\
\hline \multicolumn{13}{|c|}{ Members with $E W(\mathrm{H} \alpha)>100 \AA$} \\
\hline 557 & 11:04:05.0 & $-76: 39: 33$ & 19.84 & 17.75 & 15.95 & 12.953 & 11.757 & 10.903 & & & & M4.5 \\
\hline 562 & 11:08:03.9 & $-77: 38: 44$ & 17.61 & 16.00 & 14.63 & 11.638 & 9.671 & 8.304 & & & & $\mathrm{~K}:^{3}$ \\
\hline 567 & 11:09:48.3 & $-77: 26: 30$ & 20.08 & 17.78 & 15.91 & 12.767 & 11.228 & 10.236 & & & & $\mathrm{M}^{4}$ \\
\hline 569 & $11: 11: 11.5$ & $-76: 41: 58$ & 19.13 & 18.04 & 16.98 & 15.954 & 15.059 & 14.579 & 15.90 & 15.10 & 14.71 & K7: \\
\hline 574 & $11: 16: 03.7$ & $-76: 24: 53$ & 18.97 & 17.87 & 17.16 & 15.804 & 14.973 & 14.606 & 15.70 & 14.94 & 14.43 & $\mathrm{~K}:$ \\
\hline \multicolumn{13}{|c|}{ Non members } \\
\hline 555 & 11:02:16.2 & $-77: 11: 00$ & 17.89 & 16.54 & 15.08 & 13.549 & 12.961 & 12.652 & & & & $\mathrm{M} 3.5 \mathrm{Ve}$ \\
\hline 556 & $11: 02: 52.5$ & $-77: 17: 11$ & 18.70 & 17.28 & 15.75 & 14.109 & 13.586 & 13.211 & & & & $\mathrm{M} 4.5 \mathrm{~V}$ \\
\hline 563 & 11:08:11.0 & $-77: 15: 16$ & 18.47 & 16.91 & 15.78 & 13.559 & 12.506 & 12.182 & & & & M2III \\
\hline 573 & $11: 15: 46.2$ & $-77: 24: 49$ & 18.24 & 16.57 & 14.76 & 12.933 & 12.392 & 12.037 & 12.95 & 12.34 & 12.07 & M5.5V \\
\hline 575 & $11: 20: 01.0$ & $-76: 33: 57$ & 17.96 & 16.51 & 14.91 & 13.245 & 12.641 & 12.320 & 13.23 & 12.61 & 12.32 & M5V \\
\hline
\end{tabular}

Notes:

${ }^{1}$ ESO-H $\alpha 553$ (10:56:17.1 -76:30:54) was not observed.

${ }^{2}$ Classified as M4 by Gómez \& Mardones (2003) based on the near-IR water absorption measured on near-infrared spectra.

${ }^{3}$ Classified as M2 by Gómez \& Mardones (2003) based on the near-IR water absorption measured on near-infrared spectra.

${ }^{4}$ Classified by Gómez \& Mardones (2003) as M1.5-M3, based on two measurements of the near-IR water absorption on near-infrared spectra.

appearance of the spectrum over the 6500-9000 ̊ range. Spectral types are assigned by comparison with the atlas of late stellar spectra of Kirkpatrick et al. (1991), covering the same wavelength range. The classification accuracy is estimated to be 0.5 spectral subclasses for types M4 and later, and up to one subclass for earlier types, except for veiled spectra whose spectral features are diluted by a featureless continuum. Veiling does not appear to be significant in the wavelength range covered by our spectra for the objects plotted in Fig. 2, whose emission line spectra do not display very strong $\mathrm{H} \alpha$ emission.

The five members with $\mathrm{H} \alpha$ equivalent widths exceeding $100 \AA$ are plotted in Fig. 3. Besides the strong $\mathrm{H} \alpha$, these stars also display other prominent emission lines, both permitted and forbidden. Their spectral classification is more difficult, due both to the decreased classification accuracy yielded by the spectral range under consideration for spectral types earlier than $\mathrm{M}$, and to the possible existence of continuum veiling due to accretion, which is likely judging from the existence of other accretion signatures among the emission lines. Although the distinctive feature of these objects is the prominent emission line spectrum, important differences exist among them, to be discussed in detail in Sect. 3.5. ESO-H $\alpha 569$ and ESO-H $\alpha 574$ are by far the faintest in our sample at infrared wavelengths. Their relatively blue spectral energy distributions over the visible/near infrared range indicate that such faintness is not due to extinction. On the other hand, we note that their $R_{\mathrm{C}}$ magnitudes are similar to those of many later-type members with low-tomoderate $\mathrm{H} \alpha$ emission, showing that there is no selection effect towards very low luminosity objects with very strong emission lines. The other three objects, ESO-H $\alpha$ 557, ESO-H $\alpha$ 562, and $\mathrm{ESO}-\mathrm{H} \alpha$ 567, appear to be the most reddened ones in the sample.

Finally, four of the stars turned out not to be confirmed as emission-line objects. In three cases (ESO-H $\alpha$ 556, ESO-H $\alpha$ 573, and ESO-H $\alpha$ 575) the NaI feature at $8190 \AA$ is similar to that observed in field M-type stars (Kirkpatrick et al. 1991), suggesting that these are evolved foreground M dwarfs. Their magnitudes are also generally fainter than those of the members. A fourth object, ESO-H $\alpha$ 555, also has deep NaI absorption but displays $\mathrm{H} \alpha$ emission. The $\mathrm{H} \alpha$ equivalent width, 


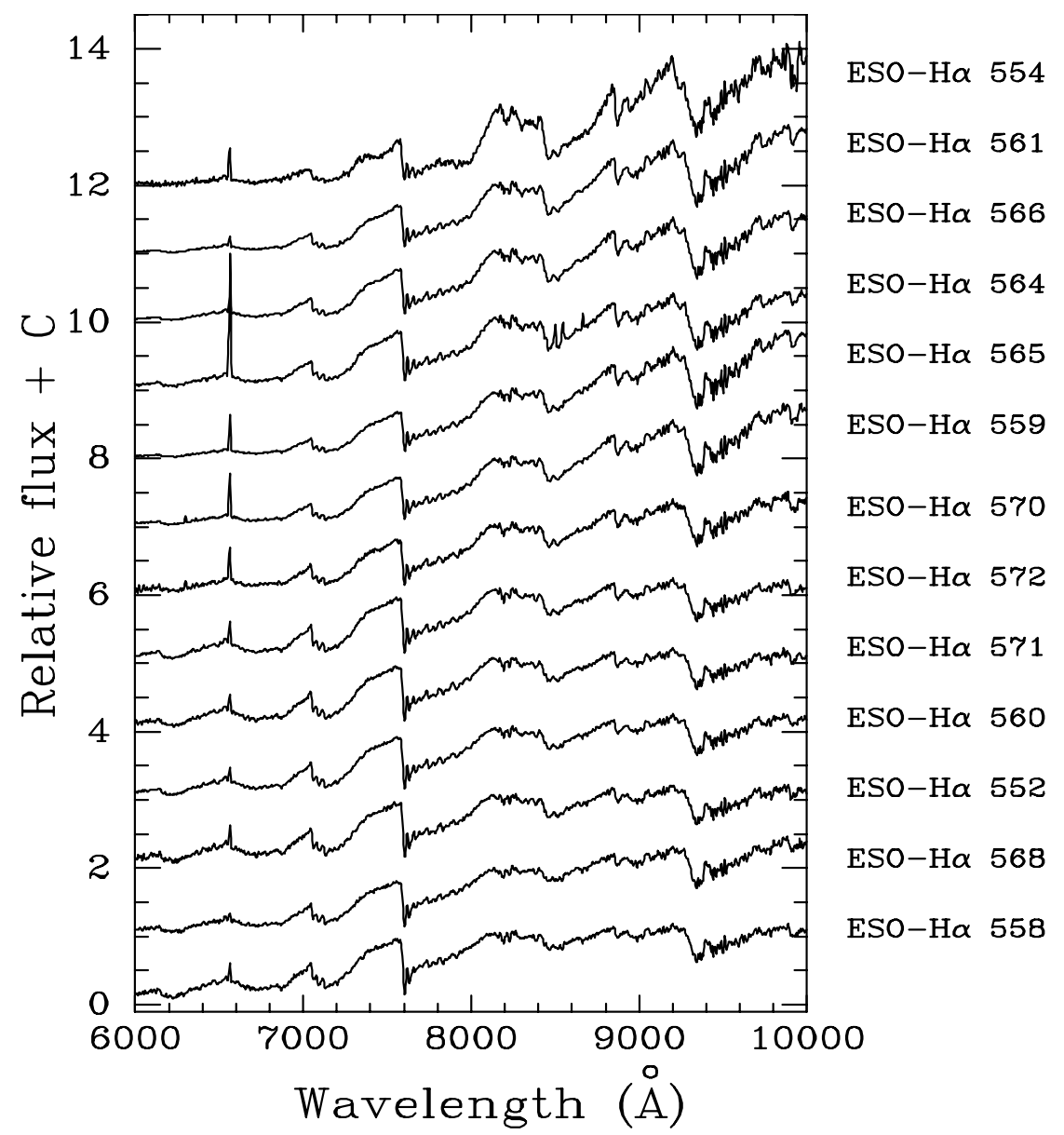

Fig. 2. Spectra of the 13 members with weak-to-moderate $\mathrm{H} \alpha$ emission identified in our sample. To facilitate comparison the spectra are arranged by order of increasing spectral type (see Table 2) from bottom to top. The strong $\mathrm{H} \alpha$ line of ESO-H $\alpha 564$ overlaps in the figure with that of ESO-H $\alpha 66$; the latter has a moderate emission with $E W(\mathrm{H} \alpha)=17.6 \AA$. All spectra are normalized to the continuum between 8000 and $9000 \AA$.

$5.1 \AA$, is small and well consistent with those observed among evolved dMe stars (e.g. Short \& Doyle 1998), in which it is attributed to chromospheric activity rather than to the accretion and outflow processes that dominate the contribution to $\mathrm{H} \alpha$ in classical $\mathrm{T}$ Tauri stars. We thus classify it as a non-member as well, although we note that its position is near that of the IRAS source B9 (Baud et al. 1984), weakly detected at $12 \mu \mathrm{m}$ only. Unfortunately, being just outside the field surveyed by ISOCAM (Persi et al. 2000) it is not possible to confirm either the flux or the positional coincidence. The fifth object that we classify as non-member, ESO-H $\alpha 563$, has an early M spectral type and no $\mathrm{H} \alpha$ emission. Some spectroscopic features, such as the relative strength of CaII absorption at $8498 \AA$ and $8542 \AA$ as compared to the NaI absorption, suggest that it is actually a M2III background giant. This is confirmed by its photometry, which indicates moderate reddening and infrared colors closer to those of giants than those of dwarfs.

In summary, we confirm 18 of our new candidates as Chamaeleon I members. Nine of them had been previously identified as possible young stellar objects on the basis of their mid-infrared emission, and two of those also because of their near-infrared colors, as discussed in Sect. 3.3. The remaining nine objects are identified for the first time in the present paper.
Table 3. Cross-identification of previously suspected members.

\begin{tabular}{ccc}
\hline \hline ESO-H $\alpha$ & Other names $^{1}$ & Reference \\
\hline 555 & BYB 9 & 1 \\
558 & BYB 18 & 1 \\
560 & ISO-ChaI 110 & 2 \\
561 & ISO-ChaI 121 & 2 \\
562 & CCE 32 & 3 \\
564 & ISO-ChaI 165 & 2 \\
565 & HJMC7-1 & 4 \\
566 & ISO-ChaI-201 & 2 \\
567 & BYB 43 & 1 \\
570 & ISO-ChaI 282 & 2 \\
\hline
\end{tabular}

Notes:

${ }^{1}$ Denominations are as in the SIMBAD database.

${ }^{2}$ Association is doubtful.

References:

(1) Baud et al. (1984); (2) Persi et al. (2000); (3) Cambrésy et al. (1998); (4) Hyland et al. (1982).

Table 3 gives other existing denominations for objects detected in previous works. 

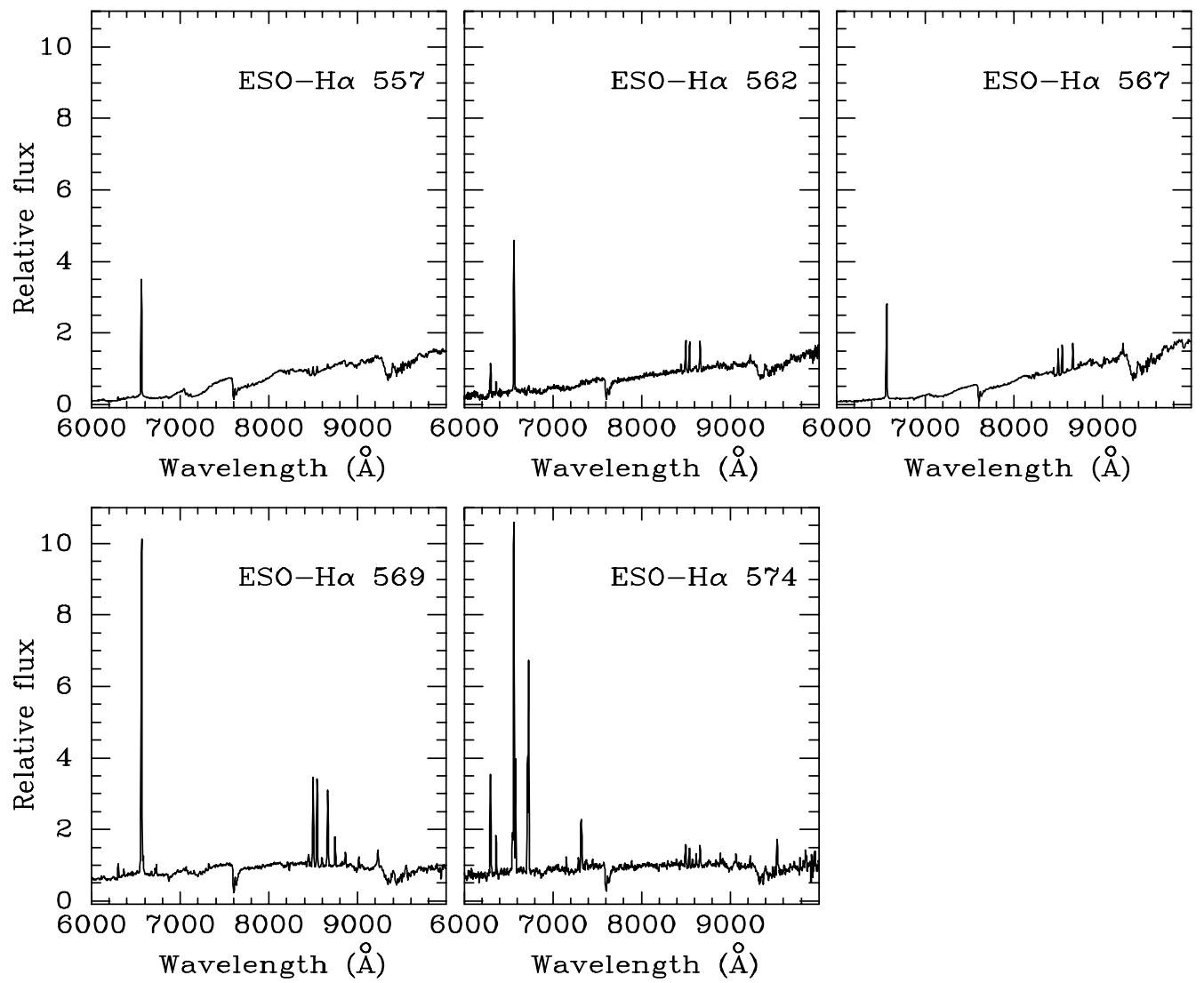

Fig. 3. Spectra of the five members observed with strong $\mathrm{H} \alpha$ emission relative to the continuum $(E W(\mathrm{H} \alpha)>100 \AA)$. The flux is normalized as in Fig. 2. To facilitate the comparison of the emission spectra among the different objects the vertical scale is the same for all the objects.

\subsection{Variability}

As noted in Table $2 J H K_{\mathrm{S}}$ photometry is available both from 2MASS and from our own observations for some objects. The differences between both are generally small and well within the combined measurement uncertainties, which we estimate at $\sim 0.1 \mathrm{mag}$. The only clear exception is ESO-H $\alpha 570$, which appears consistently brighter in all three bands in our observations by $0.50 \mathrm{mag}$ in $J, 0.43 \mathrm{mag}$ in $H$, and $0.34 \mathrm{mag}$ in $K_{\mathrm{S}}$. Its variability was already reported by Carpenter et al. (2002), whose data show amplitudes of $1.2 \mathrm{mag}$ in $J, 0.9$ mag in $H$, and $0.6 \mathrm{mag}$ in $K$ (Carpenter, priv. comm). Marginally significant differences of $0.13 \mathrm{mag}$ and $0.18 \mathrm{mag}$ in $K_{\mathrm{S}}$ are also found in $\mathrm{ESO}-\mathrm{H} \alpha 569$ and $\mathrm{ESO}-\mathrm{H} \alpha$ 574. This may be also due to the lower accuracy of our rather shallow photometry for these objects, which as noted in Sect. 3.1 are also the faintest ones in our sample in $K_{\mathrm{S}}$, the variability of ESO-H $\alpha 569$ is confirmed by monitoring observations (Carpenter, priv. comm.). An additional likely variable, based on the monitoring observations of Carpenter et al. (2002), is ESO-H $\alpha$ 567. Photometric monitoring by those authors did not detect noticeable variability in any other object of our sample, although ESO-H $\alpha 552$ and $\mathrm{ESO}-\mathrm{H} \alpha 554$ are outside the boundaries of their survey.

\subsection{Colors, extinction, and infrared excess}

The properties of our objects in visible broad-band colors are summarized in the $\left(V-R_{\mathrm{C}}\right),\left(R_{\mathrm{C}}-I_{\mathrm{C}}\right)$ diagram (Fig. 5).

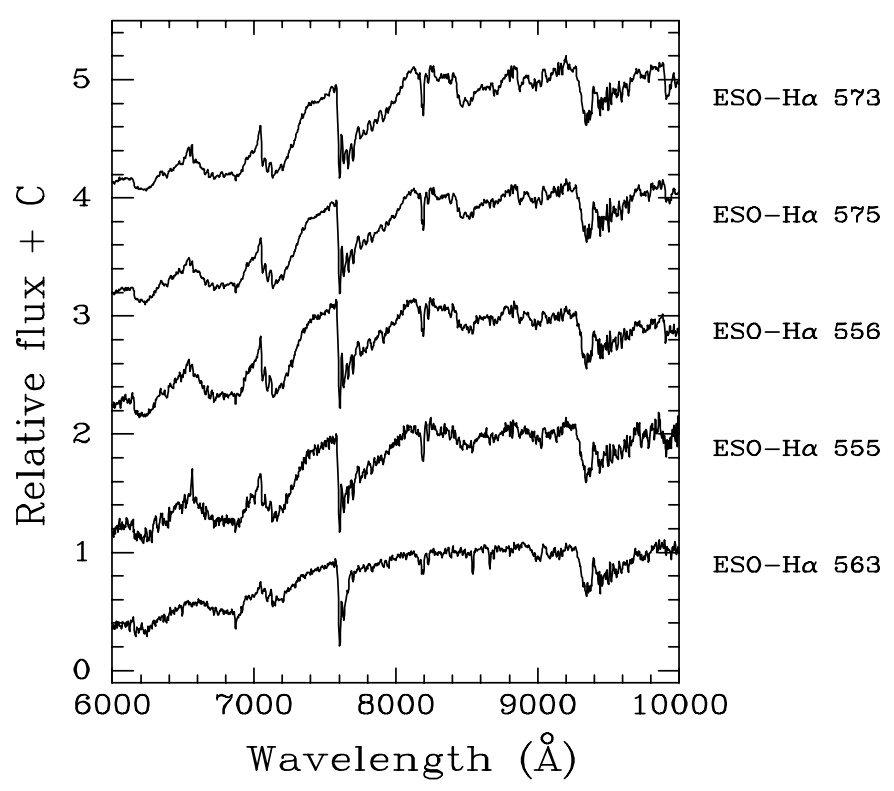

Fig. 4. Spectra of the targets that we classify as non-members. Like in Fig. 2, spectra have been arranged by order of increasing spectral type from bottom to top. Note the appearance of the gravity-sensitive NaI feature at $8190 \AA$, which is much stronger than in the spectra of the members plotted in Fig. 2.

Late-type members with weak or moderate $\mathrm{H} \alpha$ emission align along a strip that runs roughly parallel to the extinction 


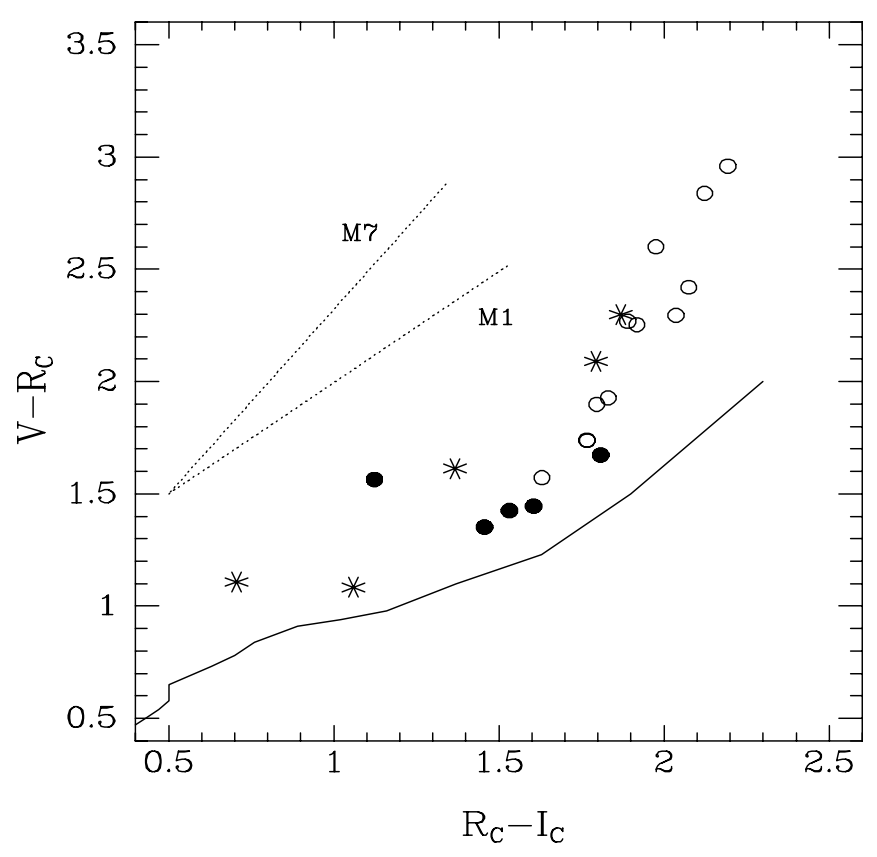

Fig. 5. Visible color-color diagram of all the objects discussed in this paper. Open circles are members with weak or moderate $\mathrm{H} \alpha$ emission, asterisks are members with $E W(\mathrm{H} \alpha)>100 \AA$, and filled circles are non-members. The solid line is the locus of main sequence stars, spanning from K1V to M6V, from Bessell (1990). The labelled dotted lines are reddening vectors for a $\mathrm{M} 1$ and a $\mathrm{M} 7$ giant obscured by $A_{\mathrm{V}}=5$, based on data by Fluks et al. (1994).

vector corresponding to a late-type photosphere (calculated from data given in Table 4 of Fluks et al. 1994 using a totalto-selective extinction ratio of $R_{V}=3.1$ ). Extinction in star forming regions, normally characterized by a larger $R_{V}$, yields a slightly steeper reddening vector (Steenman \& Thé 1989). The unreddened expected colors plotted as a reference in Fig. 5 are averages for main sequence cool dwarfs (Bessell 1990), and should be taken with caution as important systematic differences with respect to the colors of the much younger objects considered here probably exist. Indeed, color indices of a sample of over 20 M-type members of the young cluster IC 2391 by Barrado y Navascués et al. (1999), likelier to better represent the intrinsic colors of the objects discussed here, are redder by a few tenths of a magnitude in $\left(V-R_{\mathrm{C}}\right)$ and bluer by a similar amount in $\left(R_{\mathrm{C}}-I_{\mathrm{C}}\right)$ than those in the Bessell (1990) compilation. With the caveats imposed by uncertainties on intrinsic colors (and their scatter within a given spectral type; see for example Zapatero Osorio et al. 1997), and on the actual slope of the reddening vector, we conclude that the visible colors of the members with moderate $\mathrm{H} \alpha$ emission may be described by a typical late M-type, lightly reddened photosphere. Four of the non-members lie near the unreddened locus of M-type dwarfs, in agreement with their classification as foreground field stars. The fifth non-member has a distinctly redder $\left(V-R_{\mathrm{C}}\right)$ despite its early spectral type, consistent with its classification as a reddened background giant (Sect. 3.1).

Three of the sources with strong emission lines (ESO-H $\alpha$ 562, ESO-H $\alpha$ 569, and $\mathrm{ESO}-\mathrm{H} \alpha$ 574) have bluer $V-R_{\mathrm{C}}$ and $R_{\mathrm{C}}-I_{\mathrm{C}}$ colors, consistent with their earlier spectra.

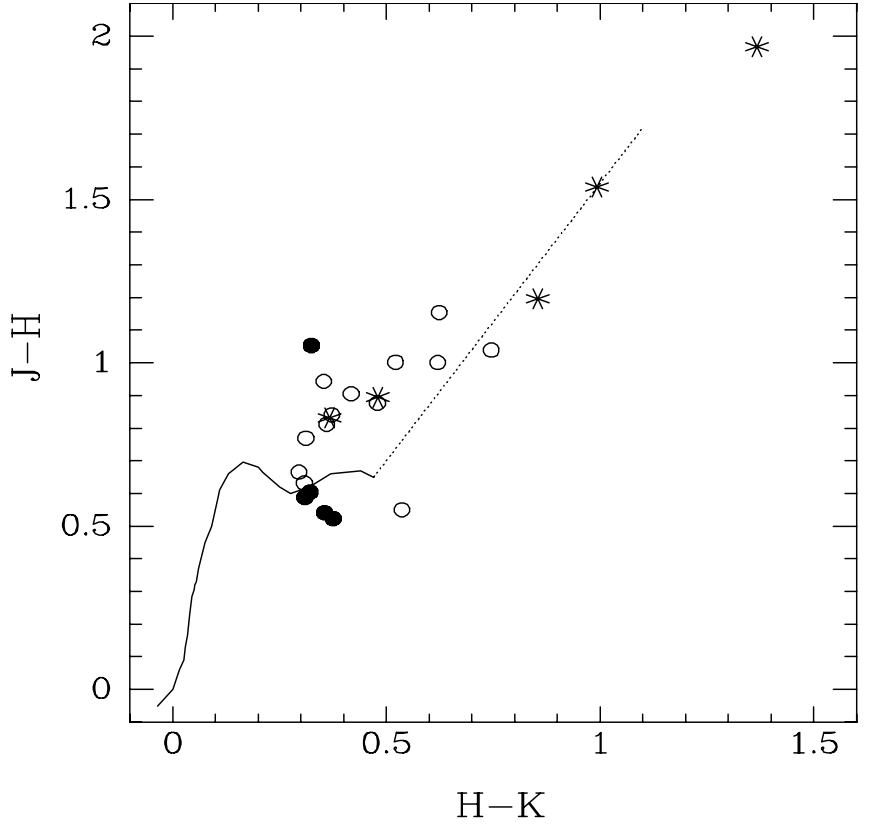

Fig. 6. Infrared color-color diagram of all the objects presented in this paper. Symbols are as in Fig. 5. The solid line is the locus of unreddened main sequence stars from Bessell \& Brett (1988). The dotted line is the reddening vector from Rieke \& Lebofsky (1985) for $A_{\mathrm{V}}=10 \mathrm{mag}$.

The contribution of the strong emission lines in the $R_{\mathrm{C}}$ band to the bluening of $R_{\mathrm{C}}-I_{\mathrm{C}}$ can be measured from our spectra and is found to be less than 0.2 mag for ESO-H $\alpha$ 574, and about $0.1 \mathrm{mag}$ for $\mathrm{ESO}-\mathrm{H} \alpha 569$ and $\mathrm{ESO}-\mathrm{H} \alpha$ 562, respectively. Continuum veiling, which appears likely in view of other accretion signs present in their spectra, and scattering by circumstellar dust are also expected to contribute to the blue colors of these objects.

In the $(J-H),\left(H-K_{\mathrm{S}}\right)$ diagram (Fig. 6) all our sources display the colors of normal photospheres reddened by different amounts without hints of strong near-infrared excesses. The members with moderate $\mathrm{H} \alpha$ emission have extinctions ranging from negligible to $A_{\mathrm{V}} \simeq 10 \mathrm{mag}$ for ESO-H $\alpha$ 567. The non-members show no indications of reddening except for the background giant ESO-H $\alpha$ 563. The position of ESO-H $\alpha 562$ at the top right of Fig. 6 seems to be mainly due to the higher extinction, but its location slightly to the right of the extension of the reddening vector may indicate a small contribution of circumstellar emission to the $K_{\mathrm{S}}$-band flux, especially taking into account that the underlying spectrum seems to be earlier than $\mathrm{M}$, thus implying a bluer intrinsic $\left(H-K_{\mathrm{S}}\right)$. Its possible excess at $K_{\mathrm{S}}$ led Cambrésy et al. (1998) to propose it as a member of Chamaeleon I. This classification has been supported both by more recent observations detecting mid-infrared emission (Kenyon \& Gómez 2001, Habart et al. 2003), and by infrared spectroscopy (Gómez \& Mardones 2003; see Table 2). Another object previously classified as a likely Chamaeleon I member is ESO-H $\alpha 565$, which was already proposed as a member of the region in the pioneering infrared work of 
Hyland et al. (1982) on the basis of its near-infrared excess ${ }^{1}$. The youth, and therefore likely membership, of this object has been subsequently confirmed by mid-infrared observations by Prusti et al. (1991), Persi et al. (2000), and Kenyon \& Gómez (2001).

As noted by Comerón et al. (2000) on a smaller sample in Chamaeleon I, while near-infrared excesses are rare or absent near the stellar/substellar boundary even for objects with strong $\mathrm{H} \alpha$ emission, mid-infrared excesses are common among them. Indeed, seven of the objects in our sample are detected in the mid infrared. Besides ESO-H $\alpha 62$ and $\mathrm{ESO}-\mathrm{H} \alpha$ 567, already noted above, ESO-H $\alpha 58$ (=B18) and ESO-H $\alpha 567$ (=B43) were detected as faint sources by IRAS at $60 \mu \mathrm{m}$ and $100 \mu \mathrm{m}$, respectively (Baud et al. 1984). Five more objects (ESO-H $\alpha$ 560, ESO-H $\alpha$ 561, ESO-H $\alpha$ 564, ESO-H $\alpha$ 566, and $\mathrm{ESO}-\mathrm{H} \alpha$ 570) have been detected by ISOCAM (Persi et al. 2000). Unfortunately, five of our sources (ESO-H $\alpha 552$, ESO-H $\alpha$ 554, ESO-H $\alpha$ 571, ESO-H $\alpha$ 572, and ESO-H $\alpha$ 574) are outside the area of the ISOCAM survey and no sensitive mid-infrared observations are available for them. Finally, only four of our sources are not detected by ISOCAM despite being within the surveyed area: ESO-H $\alpha$ 557, ESO-H $\alpha 559$, ESO-H $\alpha$ 568, and ESO-H $\alpha 69$.

\subsection{Intrinsic properties}

The spectroscopic and photometric data available for the new members of Chamaeleon I allow us to apply techniques commonly employed with other, similar objects in star forming regions to estimate masses and ages. We adopt the temperature vs. spectral type calibration proposed by Luhman et al. (1999) for very young objects with surface gravities intermediate between those of dwarfs and giants. The $J$ and $H$ bands offer the best compromise for the determination of luminosities of cool objects: they are close to the peak of their spectral energy distribution, nearly insensitive to veiling due to accretion or infrared excess due to circumstellar material, they are only moderately affected by extinction and by its dependency on grain properties, and the $(J-H)$ colors are confined to a narrow range (Bessell \& Brett 1988). Moreover, as noted in Sect. 3.2 only small amplitude variability at most is detected in most of the objects. Taking the $J$ band as a reference, we use the bolometric correction $B C_{J}$ as a function of the spectral type of Lawson et al. (1996). As discussed in Fernández \& Comerón (2001) and Comerón et al. (2003), we have adopted the spectral type vs. $(J-H)$ calibration of Bessell \& Brett (1988) as a baseline, but increasing the intrinsic color by 0.10 mag to account for apparent systematic differences between the old disk dwarfs on which Bessell \& Brett's results are based and the actual colors

\footnotetext{
1 The criterion used by Hyland et al. (1982) to discriminate between objects with and without infrared excess takes as a reference a reddening vector having its origin at the unreddened colors of the earliest type stars, i.e., the lower left end of the curve in Fig. 6, rather than the opposite end corresponding to a M6 dwarf as we do, and which thus produces a limiting reddening vector shifted to the right. Therefore our criterion to discern stars with infrared excess is somewhat more restrictive, and explains why, contrarily to Hyland et al. (1982), we do not consider ESO-H $\alpha 565$ as an infrared-excess object.
}

Table 4. Physical parameters and extinctions.

\begin{tabular}{ccccc}
\hline \hline $\begin{array}{c}\text { Object } \\
(\mathrm{ESO}-\mathrm{H} \alpha)\end{array}$ & $\begin{array}{c}\log L \\
\left(L_{\odot}\right)\end{array}$ & $A_{J}$ & $\begin{array}{c}M \\
\left(M_{\odot}\right)\end{array}$ & $\begin{array}{c}\text { Age } \\
(\mathrm{Myr})\end{array}$ \\
\hline 552 & -1.17 & 0.13 & 0.18 & 2.5 \\
554 & -2.40 & $0.00^{1}$ & 0.03 & $-^{2}$ \\
557 & -1.37 & 1.25 & 0.20 & 6 \\
558 & -1.08 & 0.24 & 0.22 & 3 \\
559 & -1.40 & 0.74 & 0.10 & 2.5 \\
560 & -1.24 & 0.32 & 0.18 & 3 \\
561 & -1.34 & 0.41 & 0.08 & 1.5 \\
564 & -1.42 & 0.74 & 0.10 & 2.5 \\
565 & -0.97 & 1.14 & 0.12 & 1 \\
566 & -1.24 & 0.49 & 0.08 & 1 \\
567 & -0.60 & 2.16 & 0.7 & 8 \\
568 & -1.03 & 0.59 & 0.22 & 2.5 \\
570 & -1.61 & 0.84 & 0.09 & 3.5 \\
571 & -1.50 & $0.00^{1}$ & 0.12 & 4 \\
572 & -1.18 & $0.00^{1}$ & 0.14 & 2 \\
\hline
\end{tabular}

Notes:

${ }^{1}$ The $(J-H)$ colors of these objects are slightly bluer than the ones assumed for their spectral types. The extinction is set to zero in these cases.

${ }^{2}$ This object is located on a region of the temperature-luminosity diagram where isochrones in the $2-5 \mathrm{Myr}$ range merge due to the temporary stability provided by deuterium burning.

of younger stars of the same spectral types. This is an ad hoc correction whose size is anyway small as compared to the intrinsic scatter in colors at a given spectral type. We have also considered the effects of using the $J H K_{\mathrm{S}}$ colors defining the T Tauri locus (1997) instead of the adopted colors based on Bessell \& Brett (1988) calibration for the dereddening of our objects. The differences found between both cases are below $0.1 \mathrm{mag}$ for all the objects in our sample. Extinction has been estimated by adopting the Rieke \& Lebofsky (1985) reddening law, and the distance to Chamaeleon I has been assumed to be $160 \mathrm{pc}$ (Wichmann et al. 1997; Whittet et al. 1997). Once the luminosity and the temperature have been obtained using all the above prescriptions, the pre-main sequence evolutionary models of Baraffe et al. (1998) have been used to derive masses and ages.

The results are summarized in Table 4, and the associated uncertainties in these parameters due to possible errors in the location of the sources in the temperature-luminosity diagram can be assessed from the error bars given in Fig. 7. We have excluded ESO-H $\alpha$ 562, ESO-H $\alpha$ 569, and ESO-H $\alpha$ 574, whose spectral types are poorly determined; the latter two are discussed separately in Sect. 3.5. Figure 7 shows the temperature-luminosity diagram of all these objects with the pre-main sequence evolutionary tracks superimposed. Most of them appear above the $5 \mathrm{Myr}$ isochrone, in agreement with the age of $2 \mathrm{Myr}$ estimated by Lawson et al. (1996) and Comerón et al. (2000). Two exceptions may be ESO-H $\alpha 57$ and ESO-H $\alpha$ 567, both having $\mathrm{H} \alpha$ equivalent widths exceeding $100 \AA$ and appearing slightly older. The error bars in Fig. 7 do not exclude an age similar to that of the other objects plotted there, but a similar behavior has also been noted by Comerón et al. (2003) among sources of the Lupus 3 cloud and may 
Table 5. Emission line equivalent widths (in $\AA$ ) of new members with $E W(\mathrm{H} \alpha)<100 \AA$.

\begin{tabular}{|c|c|c|c|c|c|c|c|c|c|c|c|c|c|}
\hline \multirow{2}{*}{$\begin{array}{c}\text { Line } \\
(\lambda \text { in } \AA)\end{array}$} & \multicolumn{13}{|c|}{ Object $(\mathrm{ESO}-\mathrm{H} \alpha)$} \\
\hline & 552 & 554 & 558 & 559 & 560 & 561 & 564 & 565 & 566 & 568 & 570 & 571 & 572 \\
\hline [OI] 6300 & & & & 11.8 & & & & & & & 13.0 & & \\
\hline [OI] 6364 & & & & 3.7 & & & & & & & & & \\
\hline $\mathrm{H} \alpha 6563$ & 9.8 & 55.9 & 6.7 & 43.6 & 7.3 & 12.7 & 91.1 & 43.3 & 17.6 & 5.7 & 19.5 & 8.5 & 10.0 \\
\hline CaII 8498 & & & & & & & 5.2 & & & & & & \\
\hline CaII 8542 & & & & & & & 4.1 & & & & & & \\
\hline CaII 8662 & & & & & & & 2.4 & & & & & & \\
\hline
\end{tabular}

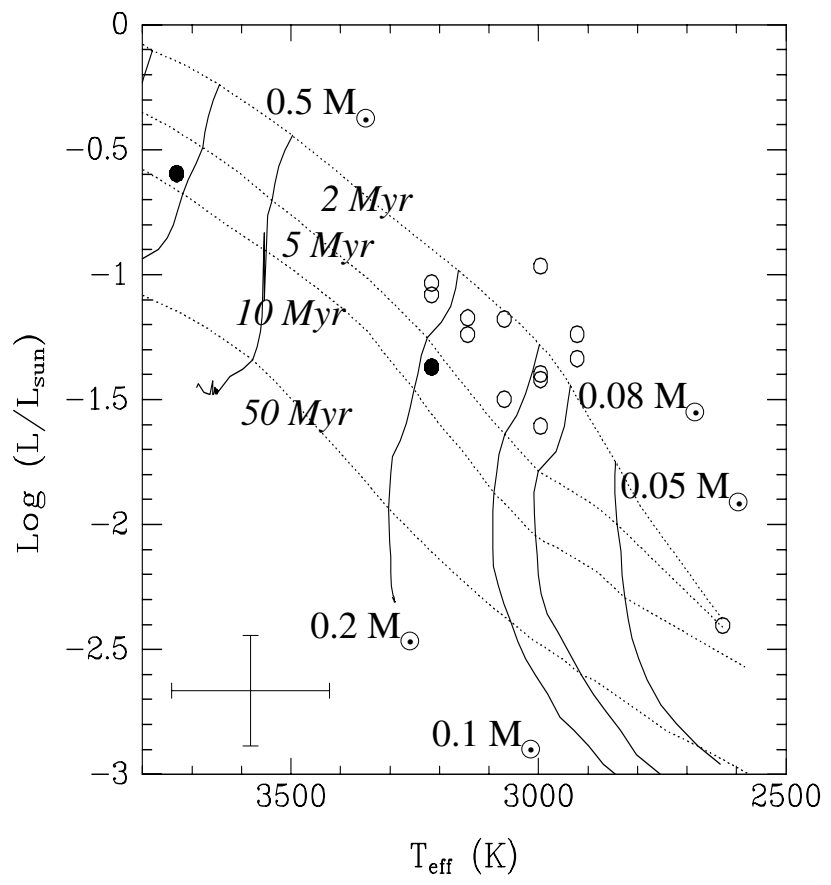

Fig. 7. Temperature-luminosity diagram of the objects listed in Table 4. The evolutionary tracks and isochrones of Baraffe et al. (1998) are indicated. Open circles correspond to objects with $E W(\mathrm{H} \alpha)<100 \AA$, filled circles to objects with $E W(\mathrm{H} \alpha)>100 \AA$. The strong emission-line objects ESO-H $\alpha$ 562, ESO-H $\alpha$ 569, and ESO$\mathrm{H} \alpha 574$ have been excluded from the diagram. The error bars given at the bottom left account for the uncertainties due to spectral misclassification by one subclass, to the scatter in the intrinsic colors assumed in the dereddening, and to photometric measurements. They do not account for systematic effects affecting all the objects in a similar way, such as offsets in the temperature-spectral type calibration, errors in the adopted distance to Chamaeleon I, or errors in the pre-main sequence models used here.

indicate a trend that manifest itself in a much more extreme way in ESO-H $\alpha 569$ and ESO-H $\alpha 574$.

The near-infrared variability properties of the two variable objects with $E W(\mathrm{H} \alpha)>100 \AA$ in our sample (Sect. 3.2) also differ from those of the only clearly variable object with $E W(\mathrm{H} \alpha)<100 \AA$. The infrared monitoring data of Carpenter et al. (2002; Carpenter, priv. comm.) for ESO-H $\alpha 567$ and ESO-H $\alpha 569$ show that both objects become redder as they brighten, a trend opposite to what is found for either hot or cold surface spots and that might point to the inner parts of the disk as the responsible for the variability. The opposite is true for ESO-H $\alpha$ 570, whose decreasing amplitude with wavelength agrees better with the behavior expected from rotationmodulated variability caused by surface spots, although the amplitudes are unusually large in the infrared. A tentative periodicity of 3.35 days is found in Carpenter's data for this object, while no periodicity is apparent from the data for the other two. Incidentally, the variability behavior of Par-Lup3-4 described by Comerón et al. (2003) is similar to that of ESO-H $\alpha$ 570, although probably smaller in amplitude, despite of its spectral characteristics and apparent underluminosity being more similar to those of ESO-H $\alpha 569$.

\subsection{Emission-line spectra and luminosities}

Most of the objects with moderate $\mathrm{H} \alpha$ equivalent widths in our survey do not display any other measurable emission lines at the resolution and signal-to-noise ratio of our spectra. Only $\mathrm{ESO}-\mathrm{H} \alpha 564$ (whose $\mathrm{H} \alpha$ equivalent width is very close to our arbitrary threshold of $100 \AA$ ) displays CaII emission, whereas faint $[\mathrm{OI}]$ emission is seen in ESO-H $\alpha 559$ and ESO-H $\alpha 570$ (Table 5).

The spectra of objects with $E W(\mathrm{H} \alpha)>100 \AA$ are richer in other emission lines characteristic of classical T Tauri stars. Forbidden lines are considered to be formed mainly in jets or winds in a region having a typical overall extent of $\sim 50 \mathrm{AU}$ (Edwards et al. 1987), and probe different conditions of density and excitation (Hamann 1994). [OI] lines are formed closest to the star, mainly due to their higher critical density, as confirmed by high spatial resolution observations (e.g. Bacciotti et al. 2000). The broad range of conditions under which they can form explains their presence in all the spectra discussed in this section. [SII] emission, having a lower critical density, is quenched in the densest regions near the star, and traces essentially the same physical conditions as [FeII] (Davis et al. 2003), while [NII] has a critical density intermediate between [OI] and [SII] and traces regions of higher excitation. Forbidden lines present distinct components with low and high velocities (Jankovics et al. 1983; Appenzeller et al. 1984; Hartigan et al. 1995) that are unfortunately indistinguishable at the resolution of our spectra. Permitted lines such as the CaII infrared triplet, HeI, and FeI are normally associated with accretion and arise from high density regions in the immediate neighbourhood of the star, either in the accretion columns where gas flows from the magnetically disrupted disk, or at the terminal shocks produced on the surface of the star itself (e.g. Königl 1991; Gullbring 1994; Edwards et al. 1994; 
Table 6. Emission line equivalent widths (in $\AA$ ) of new members with $E W(\mathrm{H} \alpha)>100 \AA$.

\begin{tabular}{|c|c|c|c|c|c|}
\hline \multirow{2}{*}{$\begin{array}{c}\text { Line } \\
(\lambda \text { in } \AA)\end{array}$} & \multicolumn{5}{|c|}{ Object $(\mathrm{ESO}-\mathrm{H} \alpha)$} \\
\hline & 557 & 562 & 567 & 569 & 574 \\
\hline [OI] 6300 & 6.3 & 41.8 & 3.7 & 6.9 & 36.5 \\
\hline [OI] 6364 & 2.8 & 8.9 & 2.9 & 2.5 & 13.3 \\
\hline FeI 6411 & 2.0 & & & & \\
\hline FeI 6472 & 2.4 & & & & \\
\hline [NII] 6548 & & & wing to $\mathrm{H} \alpha$ & wing to $\mathrm{H} \alpha$ & 13: \\
\hline $\mathrm{H} \alpha 6563$ & 149.0 & 109.8 & 177.8 & 144.1 & 131.1 \\
\hline [NII] 6583 & & 1.7: & wing to $\mathrm{H} \alpha$ & $6.7:$ & 40.7 \\
\hline HeI 6678 & 1.5 & & 1.8 & 2.4 & \\
\hline [SII] 6716 & & & & 2.4 & 42.7 \\
\hline [SII] 6730 & & & & 3.8 & 70.4 \\
\hline HeI 7066 & & & & 1.2 & \\
\hline [FeII] 7155 & & & & & 3.8 \\
\hline [CaII] 7291 & & & & & 3.0 \\
\hline [CaII] 7324 & & & & 2.4 & 26.0 \\
\hline [FeII] 7388 & & & & & 5.2 \\
\hline$[\mathrm{FeII}] 7452$ & & & & & 2.6 \\
\hline OI 8446 & 2.0 & 2.3 & 2.0 & 3.5 & \\
\hline CaII 8498 & 3.8 & 9.4 & 8.3 & 31.6 & 7.6 \\
\hline CaII 8542 & 2.2 & 8.8 & 8.5 & 31.6 & 7.2 \\
\hline FeI $8577^{1}$ & & & & & 2.6 \\
\hline Pa14 8598 & & & 0.5 & 3.3 & \\
\hline [FeII] 8617 & & & & & 3.6 \\
\hline CaII 8662 & 1.8 & 10.4 & 7.7 & 28.0 & 6.8 \\
\hline $\mathrm{Pa} 128750$ & & & 1.2 & $9.3^{2}$ & \\
\hline Pa11 8863 & & 2.2 & 1.6: & 5.6 & \\
\hline [FeII] 8892 & & & & & 3.0 \\
\hline Pa10 9015 & & 0.9: & 1.9 & 4.1 & \\
\hline [SIII] 9069 & & & & & 2.9 \\
\hline Pa9 9229 & & 2.1 & 2.3 & 6.8 & 3.6 \\
\hline [SIII] 9532 & & & & & 17.9 \\
\hline
\end{tabular}

Notes:

${ }^{1}$ Identification doubtful; other possible species are SiI and TiI (Coluzzi 1999).

${ }^{2}$ Possibly blended with FeI.

Lamzin 1995; Muzerolle et al. 1998; Beristain et al. 1998; Alencar \& Basri 2000; Alencar et al. 2001). Also $\mathrm{H} \alpha$ emission is normally linked to accretion, although other scenarios, including the bases of jets and disk winds, can also give rise to it.

Most of the spectra of our strong $\mathrm{H} \alpha$ emitters are dominated by accretion signatures, mainly $\mathrm{H} \alpha$ and the CaII triplet. The lines of high transitions of the Paschen series are also seen in the spectra of all the objects except ESO-H $\alpha 557$. We cannot accurately determine their equivalent widths, but ESO-H $\alpha 569$ stands out in the sample both because of the strength of these lines and because of their slow decrement towards higher transitions as compared to the expectation from optically thin lines in a gas at $T \simeq 10000 \mathrm{~K}$ (Brocklehurst 1971), suggesting that they are formed in a high-density, optically thick medium close to the surface of the star (Eislöffel et al. 1990). HeI is seen in three objects, probably arising from the hottest regions of the accretion columns where temperatures exceed $20000 \mathrm{~K}$ (Alencar \& Basri 2000; Beristain et al. 2001), and again it is most prominent in ESO-H $\alpha 569$.
ESO-H $\alpha 574$ presents interesting differences from the rest in both outflow and accretion signatures. Its forbidden lines are both more numerous and intense, including [FeII] lines that are not seen in the spectra of the other objects, while the CaII permitted lines are moderately intense and HeI emission is absent. Furthermore, its spectrum displays two prominent lines of [CaII], especially at $7324 \AA$, which is much more intense than the CaII triplet components, suggesting that CaII emission in this object takes place under conditions of much lower density than for the others. Unlike those of the other objects, the spectrum of ESO-H $\alpha 574$ is thus dominated by outflow tracers.

The luminosities of ESO-H $\alpha 569$ and ESO-H $\alpha 574$ would make them fall below the main sequence in Fig. 7 if derived in the same way as outlined in Sect. 3.4: assuming an intrinsic $(J-H) \simeq 0.72$, we obtain luminosities of $\log L\left(L_{\odot}\right) \simeq-2.5$ for both objects. We obtain in the same way $\log L\left(L_{\odot}\right) \simeq 0.5$ for $\mathrm{ESO}-\mathrm{H} \alpha$ 562, but given the uncertainty in its spectral type it is unclear if this implies a lower-than-expected luminosity as well. 
In view of the nearly identical photometric characteristics of ESO-H $\alpha 569$ and ESO-H $\alpha$ 574, their similar degrees of underluminosity with respect to other young members of Chamaeleon I with similar spectral types, and their similar continua, the differences between their emission line spectra are particularly intriguing. As noted above, ESO-H $\alpha 69$ is the object showing the strongest signatures of accretion with only weak outflow indicators, while the opposite is true for ESO-H $\alpha$ 574. Possible explanations for the apparent underluminosity of young stellar objects with strong $\mathrm{H} \alpha$ equivalent widths have been discussed by Comerón et al. (2003) based on a sample of very low mass objects in the Lupus 3 clouds, as well as an additional object in R CrA reported by Fernández \& Comerón (2001). As noted in Comerón et al. (2003), the actual cause for the prominence of $\mathrm{H} \alpha$ is an unusually low level of the continuum rather than the absolute intensity of that line, which is found to be similar or even below that of other objects with normal luminosity. The two most relevant cases in their sample, Par-Lup3-4 and LS-RCrA 1, are bracketed now by ESO-H $\alpha 56$ and ESO-H $\alpha$ 574. Both Par-Lup3-4 and LS-RCrA 1 show $E W(\mathrm{H} \alpha) \sim 400 \AA$, but this value is very sensitive to the level of veiling at $\mathrm{H} \alpha$, which is likely to affect more the Chamaeleon I objects. However, both the accretion signatures of ESO-H $\alpha 569$ and the outflow signatures of ESO-H $\alpha 574$ are more prominent than the corresponding ones of Par-Lup3-4 and LS-RCrA 1.

Line spectra similar to that of ESO-H $\alpha 574$ are found for some late K or early M stars, e.g. Haro 6-13, LkH $\alpha$ 188/G4 and LDN 1448 IRS 1. Both Haro 6-13 (Strom et al. 1986) and LDN 1448 IRS 1 (Eislöffel 2000) are likely outflow-driving sources. All three sources have $V$ magnitudes far too weak, ranging from 17.7 to $19.5 \mathrm{mag}$, considering their spectral types and assigned distances. Besides the strong Balmer line emission (Cohen \& Kuhi 1979), the only forbidden lines expected in the wavelength interval covered by Cohen \& Kuhi and at the resolution of their spectra are the [OI] 6300 and 6360 lines, which are clearly detected in these objects.

Comerón et al. (2003) favored intrinsic underluminosity as the explanation to the features of Par-Lup3-4 and LS-RCrA 1, probably caused by accretion-modified pre-main sequence evolution (Hartmann et al. 1997) at very low masses, as opposed to a geometric effect resulting from the occultation of the central object by an edge-on disk. In the latter case, the spectrum of the central object would be seen through light scattered by dust, thus explaining both the faintness and the nearly normal colors. The arguments put forward by Comerón et al. (2003) are based on the visibility of accretion indicators that, being formed near the surface of the object, should also be similarly occulted by an edge-on disk. Those arguments may not apply to ESO-H $\alpha$ 574, whose accretion signatures are subdued with respect to the forbidden lines formed in an extended volume that would be largely unobscured by the accretion disk. We thus consider that the edge-on disk explanation may account for the emission line spectrum and the underluminosity of ESO-H $\alpha$ 574. However, the opposite is true for ESO-H $\alpha 569$, whose far more prominent accretion signatures as compared to Par-Lup3-4 and LS-RCrA 1 reinforce the arguments given by Comerón et al. (2003) in favor of intrinsic underluminosity.
Furthermore, the non-detection of ESO-H $\alpha 569$ by ISOCAM (Persi et al. 2000) suggests that the amount of warm dust associated with this object is small. This supports that the faintness of this object in the red/near infrared is not due to occultation by circumstellar material, since a disk massive enough to block the direct light from a normal central pre-main sequence K star (and to provide the dusty medium that scatters light towards us) should be prominent in the thermal infrared. Taking into account that both the $\mathrm{H} \alpha$ fluxes and the inferred accretion rates for such objects are small in absolute terms in the intrinsic underluminosity scenario (Comerón et al. 2003), the non-detection at thermal wavelengths becomes easier to explain. Unfortunately, the lack of ISOCAM observations of ESO-H $\alpha 574$ prevents a comparison between the thermal infrared properties of these two objects which could provide useful insights on possible intrinsic differences.

In summary, we believe that $\mathrm{ESO}-\mathrm{H} \alpha 569$ provides additional support for the interpretation of some young stellar objects with large $\mathrm{H} \alpha$ equivalent widths as intrinsically underluminous objects. We are less certain concerning ESO-H $\alpha$ 574, for which an edge-on disk could also be a valid explanation. It is puzzling however that, if two independent explanations account for the apparent underluminosity of ESO-H $\alpha 569$ and ESO-H $\alpha$ 574, they turn out to produce two objects with nearly identical spectral energy distributions and degrees of underluminosity.

\subsection{Comments on techniques for the identifications of members}

Given the ubiquity of $\mathrm{H} \alpha$ emission among young stellar and substellar objects, methods for the selection of candidates based on this feature are well suited to provide a highly complete census of star forming regions, as demonstrated by the 9 new members of the Chamaeleon I presented in this paper that had not been identified so far by any of the existing alternative approaches. As discussed by Carpenter et al. (2002), infrared surveys based on IJK colors (Cambrésy et al. 1998), $J H K$ colors, or near-infrared variability (Carpenter et al. 2002) miss a sizeable fraction of the members identified by other criteria. They are nevertheless effective in allowing the survey of large areas with a reasonable investment of time in relatively small telescopes, and have the advantage of probing also the more heavily obscured regions of the star forming cloud. The drawbacks of infrared color-selected or variability-selected samples are exemplified by the objects described in the present paper, none of which displays an obvious $K$-band excess and only one of which is clearly identified as variable. On the other hand, the objective prism observations in which the sources discussed in this paper were discovered have proved to be sensitive enough to reveal new members with modest levels of $\mathrm{H} \alpha$ emission, as 6 of the new members have $E W(\mathrm{H} \alpha)<10 \AA$ (a seventh object also with $E W(\mathrm{H} \alpha)<10 \AA$ is classified as non-member in Sect. 3.1). This sensitivity to small $\mathrm{H} \alpha$ equivalent widths comes at the cost of a number of false detections in which $\mathrm{H} \alpha$ emission is not confirmed by follow-up long-slit spectra (4 out of our 23 objects). 
Sensitive mid-infrared observations such as those carried out by ISOCAM closely compete with $\mathrm{H} \alpha$ surveys in detecting members previously unrecognized by other means. The complementarity of both methods is illustrated by the fact that four of the sources that we found in the area common to the ISOCAM survey of Persi et al. (2000) are not detected in the mid-infrared (including the particularly interesting object ESO-H $\alpha$ 569), while a number of ISOCAM sources remain unidentified by any other selection criteria, including $\mathrm{H} \alpha$ emission.

An alternative technique that takes advantage of the usefulness of $\mathrm{H} \alpha$ emission as a membership identifier is provided by narrow-band imaging centered on $\mathrm{H} \alpha$ combined with broadband photometry, as recently attempted by López Martí (2003) and López Martí et al. (2003). Indeed, 13 of our H $\alpha$-emitting objects are included in the area surveyed by López Martí (2003), and 8 of them are recognized as members in that work. In three of the remaining five cases, the $\left(R_{\mathrm{C}}-I_{\mathrm{C}}\right)$ color that we measure is outside or very near the edge of the broad-band color selection range imposed by López Martí for the selection of her late-type candidates, explaining the exclusion from her list. Only two objects that we would have expected to be present in the list of candidate members of López Martí are missing, ESO-H $\alpha 557$ and $\mathrm{ESO}-\mathrm{H} \alpha$ 560. The absence of the latter can be explained by its modest level of $\mathrm{H} \alpha$ emission $(E W(\mathrm{H} \alpha)=7.3 \AA)$. This does not seem to be the case for the former, but the possibility that it might have fallen in one of the gaps of the detector mosaic used by López Martí might provide an explanation.

Despite the identification of a new brown dwarf with spectral type M8.5, which is the latest spectroscopically confirmed brown dwarf reported in Chamaeleon I so far, there is an obvious lack of objects later than M6.5 in our sample due to progressive incompleteness towards later spectral types, rather than to an actual scarcity of such cool objects. Indeed, the deeper slitless spectroscopy observations of Comerón et al. (2000) on the central region of the complex identified 6 objects with spectral types later than M6.5, of which 3 are recovered by our observations. These include Cha $\mathrm{H} \alpha 1$ and $\mathrm{Cha} \mathrm{H} \alpha 6$, which are the strongest $\mathrm{H} \alpha$ emitters in Comerón et al.'s sample. Moreover, a large number of late M and early L-type brown dwarfs is suggested by the $R I \mathrm{H} \alpha$ photometry of López Martí (2003). Since the incompleteness for the latest objects becomes a rather complicated function of the object brightness, its $\mathrm{H} \alpha$ emission, the foreground extinction, and also to some extent of a subjective factor due to the visual selection of $\mathrm{H} \alpha$-emitting objects in the Schmidt films, we refrain from using the findings reported in this paper to draw consequences on the initial mass function of the star forming region.

\section{Summary and conclusions}

In this paper we have presented the results of a deep objective prism survey of the entire Chamaeleon I star forming region aiming at the detection of new faint members with $\mathrm{H} \alpha$ emission. Our objective prism films reveal 24 new objects for which no previous spectroscopic observations in the visible were available. We have performed low-resolution long slit spectroscopy of all but one of them, confirming 18 as members and discarding 5 as either foreground stars or background giants. Half of the members were already suspected previous to this work based on other signatures of youth, mainly thermal infrared excess. The other half is identified as new members for the first time in this work. The spectral types of the new members range from late $\mathrm{K}$ to M8.5, although we suspect serious incompleteness of our sample for types later than M6.

The visible colors of all the members are consistent with reddened photospheres, possibly with continuum veiling in some of them. In the near-infrared, none of our objects displays obvious signatures of $K$-band excess of circumstellar origin. Three objects are variable based on previously existing nearinfrared monitoring data, although with important differences among them concerning their variability properties.

The accurate spectral types obtained in this paper for most of the members complemented with 2MASS infrared photometry allow us to estimate their ages and masses using theoretical pre-main sequence evolutionary tracks. We find ages between 1 and $5 \mathrm{Myr}$ for most objects, consistent with previous determinations of the age of the aggregate. Their masses are mostly above or near the hydrogen-burning limit, except for the latesttype object identified in our sample, whose M8.5 spectral type indicates a brown dwarf mass of $0.03 M_{\odot}$.

Two rather exceptional objects are found with a rich emission line spectrum and very low luminosity at infrared wavelengths. While they have almost identical broad-band spectral energy distributions, the details of their emission-line spectra are very different. One of them displays very prominent permitted lines that are normally attributed to accretion, while the other is dominated by outflow signatures in the form of forbidden lines, such as those generally observed in jets and winds. Other objects also having rich emission line spectra and very low luminosity have been identified in other regions. We propose that the accretion-signature-dominated emission spectrum of one of these objects, as well as its non-detection in the thermal infrared, supports the interpretation of the apparent underluminosity as being an intrinsic feature of the object rather than the result of a special viewing geometry in which a normal luminosity object is blocked from direct view by an edge-on disk and is seen only in scattered light. It is doubtful that the same explanation applies to the second object, whose emission spectrum, dominated by outflow signatures, would be far less blocked by an edge-on disk.

We confirm deep $\mathrm{H} \alpha$ surveys as a most competitive way to sample the low-luminosity population of lightly obscured regions of the nearest star forming clouds. A comparison to other member selection criteria confirms that most cool $\mathrm{H} \alpha$ emitting objects do not display either noticeable infrared excesses or variability, thus showing the advantage of the $\mathrm{H} \alpha$ selection criterion in providing a highly complete census of members. Only the sensitive ISOCAM data seem to produce similarly complete samples at such low luminosities.

No extended areas of high extinction such as those existing in star forming regions like $\rho$ Ophiuchi are observed in Chamaeleon I, thus ruling out the possible existence of a heavily obscured population inaccessible to observations in the 
visible range. This, together with the fact that $\mathrm{H} \alpha$ emission is the most common feature among young stellar objects, leads us to suspect that the members newly identified in this paper represent an important step forward towards a complete census of the entire Chamaeleon I population reaching down to the most massive brown dwarfs, thus opening the door to studies of its collective properties minimally affected by selection biases. Some caveats need however to be solved before statistical conclusions about the lowest mass component can be conclusively drawn: the completeness limits of a $\mathrm{H} \alpha$ survey is a function combining the object magnitude, the $\mathrm{H} \alpha$ equivalent width, and the foreground extinction that needs to be carefully taken into account. Comparison with deeper slitless spectroscopy surveys over smaller areas or with narrow-band $\mathrm{H} \alpha$ imaging observations indeed confirm that the census of $\mathrm{H} \alpha$-emitting members is still very incomplete beyond spectral type M6.5 at the least. On the other hand, there are a number of objects detected at midinfrared wavelengths that remain undetected in this $\mathrm{H} \alpha$ survey. Once the nature of all the sources identified by any technique is clarified, and robust determinations of their intrinsic properties (mass, age, evolutionary status) are available, the analysis of the global properties of the entire population of Chamaeleon I can proceed on a firm standing. Given the rapid progress in this direction provided by the various observational projects currently in progress, this goal is probably within reach in the near future.

Acknowledgements. We are grateful to Guido and Oscar Pizarro, who as telescope operators obtained all the Schmidt films used in this survey. The support of the La Silla Observatory during our observing run at the NTT, especially Dr. Emanuela Pompei, Mr. Duncan Castex, and Ms. Mónica Castillo, is warmly acknowledged. We are also very pleased to thank Dr. John Carpenter for kindly making available to us the data obtained for his variability monitoring project on Chamaeleon I. M.F. is partially supported by the Spanish grant PB97-1438-C02-02. This publication makes use of data products from the Two Micron All Sky Survey, which is a joint project of the University of Massachusetts and the Infrared Processing and Analysis Center/California Institute of Technology, funded by the National Aeronautics and Space Administration and the National Science Foundation. This research has made use of the VizieR catalogue service and the SIMBAD database, operated at CDS, Strasbourg, France. The Digitized Sky Survey was produced at the Space Telescope Science Institute under US Government grant NAG W-2166. The images of these surveys are based on photographic data obtained using the Oschin Schmidt Telescope on Palomar Mountain and the UK Schmidt Telescope. The plates were processed into the present compressed digital form with the permission of these institutions.

\section{References}

Alcalá, J. M., Krautter, J., Schmitt, J. H. M. M., et al. 1995, A\&AS, 114,109

Alcalá, J. M., Krautter, J., Covino, E., et al. 1997, A\&A, 319, 184

Alencar, S. H. P., \& Basri, G. 2000, AJ, 119, 1881

Alencar, S. H. P., Johns-Krull, C. M., \& Basri, G. 2001, AJ, 122, 3335

Apai, D., Pascucci, I., Henning, Th., et al. 2002, ApJ, 573, L115

Appenzeller, I., Jankovics, I., \& Östreicher, R. 1984, A\&A, 141, 108

Bacciotti, F., Mundt, R., Ray, T.P., et al. 2000, ApJ, 537, L49
Baraffe, I., Chabrier, G., Allard, F., \& Hauschildt, P. H. 1998, A\&A, 337,403

Barrado y Navascués, D., Stauffer, J. R., \& Patten, B. M. 1999, ApJ, 522, L53

Baud, B., Beintema, D. A., Wesselius, P. R., et al. 1984, ApJ, 278, L53

Béjar, V. J. S., Zapatero Osorio, M. R., \& Rebolo, R. 1999, ApJ, 521, 671

Beristain, G., Edwards, S., \& Kwan, J. 1998, ApJ, 499, 828

Beristain, G., Edwards, S., \& Kwan, J. 2001, ApJ, 551, 1037

Bessell, M. S. 1990, A\&AS, 83, 357

Bessell, M. S., \& Brett, J. M. 1988, PASP, 100, 1134

Brocklehurst, M. 1971, MNRAS, 153, 417

Cabrit, S., Edwards, S., Strom, S. E., \& Strom, K. M. 1990, ApJ, 354, 687

Cambrésy, L., Copet, E., Epchtein, N., et al. 1998, A\&A, 338, 977.

Carpenter, J. M., Meyer, M. R., Dougados, C., Strom, S. E., \& Hillenbrand, L. A. 1997, AJ, 114, 198.

Carpenter, J. M., Hillenbrand, L. A., Skrutskie, M. F., \& Meyer, M. R. 2002, AJ, 124, 1001

Chen, H., Grenfell, T. G., Myers, P. C., \& Hughes, J. D. 1997, ApJ, 478, 295

Cohen, M., \& Kuhi, L. V. 1979, ApJS, 41743

Coluzzi, R. 1999, revised version of Coluzzi, R. 1993, Bull. Inf. CDS, 43, 7 (available through VizieR On-line Data Catalogs)

Comerón, F., Rieke, G. H., \& Neuhäuser, R. 1999, A\&A, 343, 477

Comerón, F., Neuhäuser, R., \& Kaas, A. A. 2000, A\&A, 359, 269

Comerón, F., Fernández, M., Baraffe, I., Neuhäuser, R., \& Kaas, A. A. 2003, A\&A, 406, 1001

Covino, E., Alcala, J. M., Allain, S., et al. 1997, A\&A, 328, 187

Davis, C. J., Whelan, E., Ray, T. P., \& Chrysostomou, A. 2003, A\&A, 397, 693

Edwards, S., Cabrit, S., Strom, S.E., et al. 1987, ApJ, 321, 473

Edwards, S., Hartigan, P., Ghandour, L., \& Andrulis, C. 1994, AJ, 108, 1056

Eislöffel, J. 2000, A\&A, 354, 236

Eislöffel, J., Solf, J., \& Böhm, K. H. 1990, A\&A, 237, 369

Fernández, M., \& Comerón, F. 2001, A\&A, 380, 264

Fluks, M. A., Plez, B., Thé, P. S., et al. 1994, A\&AS, 105, 311

Gómez, M., \& Kenyon, S. J. 2001, AJ, 121, 2673

Gómez, M., \& Mardones, D. 2003, AJ, 125, 2134

Gómez, M., \& Persi, P. 2002, A\&A, 389, 494

Gorlova, N., Rieke, G. H., \& Liebert, J. 2003, ApJ, 593, 1074

Greene, T. P., \& Meyer, M. R. 1995, ApJ, 450, 233

Greene, T. P., \& Lada, C. J. 1996, AJ, 112, 2184

Gullbring, E. 1994, A\&A, 287, 131

Gürtler, J., Schreyer, K., Henning, Th., Lemke, D., \& Pfau, W. 1999, A\&A, 346, 205

Habart, E., Testi, L., Natta, A., \& Vanzi, L. 2003, A\&A, 400, 575

Hamann, F., \& Persson, S. E. 1992, ApJ, 394, 628

Hamann, F. 1994, ApJS, 93, 485

Hamuy, M., Walker, A. R., Suntzeff, N. B., et al. 1992, PASP, 104, 533

Hartigan, P. 1993, AJ, 105, 1511

Hartigan, P., Edwards, S., \& Ghandour, L. 1995, ApJ, 452, 736

Hartmann, L., Cassen, P., \& Kenyon, S. J. 1997, ApJ, 475, 770

Henize, K. G., \& Mendoza, E. E. 1973, ApJ, 180

Huenemoerder, D. P., Lawson, W. A., \& Feigelson, E. D. 1994, MNRAS, 271, 967

Hyland, A. R., Jones, T. J., \& Mitchell, R. M. 1982, MNRAS, 201, 1095

Jankovics, I., Appenzeller, I., \& Krautter, J. 1983, PASP, 95, 883

Joergens, V., \& Guenther, E. 2001, A\&A, 379, L9

Joergens, V., Fernández, M., Carpenter, J. M., \& Neuhäuser, R. 2003, ApJ, 594, 971 
Kenyon, S. J., \& Gómez, M. 2001, AJ, 121, 2673

Kirkpatrick, J. D., Henry, T. J., \& McCarthy, D. W., 1991, ApJS, 77, 417

Königl, A. 1991, ApJ, 370, L39

Lamzin, S. A. 1995, A\&A, 295, L20

Landolt, A. U. 1992, AJ, 104, 340

Lawson, W. A., Feigelson, E. D., \& Huenemoerder, D. P. 1996, MNRAS, 280, 1071

López Martí, B. 2003, Ph.D. Thesis, Univ. of Valencia

López Martí, B., Eislöffel, J., \& Mundt, R. 2003, A\&A, submitted

Luhman, K. L., Rieke, G. H. 1998, ApJ, 497, 354

Luhman, K. L. 1999, ApJ, 525, 466

Martín, E. L., Rebolo, R., \& Zapatero Osorio, M. R. 1996, ApJ, 469, 706

Martín, E. L., Basri, G., \& Zapatero Osorio, M. R. 1999, AJ, 118, 1005

Meyer, M. R., Calvet, N., \& Hillenbrand, L. A. 1997, AJ, 114, 288

Muzerolle, J., Calvet, N., \& Hartmann, L. 1998, ApJ, 492, 743

Natta, A., \& Testi, L. 2001, A\&A, 376, L22

Natta, A., Testi, L., Comerón, F., et al. 2002, A\&A, 393, 259

Neuhäuser, R., \& Comerón, F. 1998, Science, 282, 83

Neuhäuser, R., \& Comerón, F. 1999, A\&A, 350, 612

Neuhäuser, R., Brandner, W., Alves, J., Joergens, V., \& Comerón, F. 2002, A\&A, 384, 999
Oasa, Y., Tamura, M., \& Sugitani, K. 1999, ApJ, 526, 336

Persi, P., Marenzi, A. R., Kaas, A. A., et al. 1999, AJ, 117, 439

Persi, P., Marenzi, A. R., Olofsson, G., et al. 2000, A\&A, 357, 219

Persson, S. E., Murphy, D. C., Krzeminski, W., Roth, M., \& Rieke, M. J. 1998, AJ, 116, 2475

Pettersson, B., \& Reipurth, B. 1994, A\&A Suppl, 104, 233

Prusti, T., Clark, F. O., Whittet, D. C. B., Laureijs, R. J., \& Zhang, C. Y. 1991, MNRAS, 251, 303

Reipurth, B. 1996, Messenger, 85, 8

Reipurth, B., Pettersson, B., Armond, T., Bally, J., \& Vaz, L. P. R. 2003, AJ, in press

Rieke, G. H., \& Lebofsky, M. J. 1985, ApJ, 288, 618

Schwartz, R. D. 1977, ApJS, 35, 161

Schwartz, R. D. 1991, in Low Mass Star Formation in Southern Molecular Clouds, ed. B. Reipurth, ESO Scientific Report No. 11

Short, C. I., \& Doyle, J. G. 1998, A\&A, 336, 613

Steenman, H. C., \& Thé, P. S. 1989, Ap\&SS, 159, 189

Strom, K. M., Strom, S. E., Wolff, S. C., Morgan, J., \& Wenz, M. 1986, ApJS, 62, 39

Whittet, D. C. B., Prusti, T., Franco, G. A. P., et al. 1997, A\&A, 327, 1194

Wichmann, R., Krautter, J., Covino, E., et al. 1997, A\&A, 320, 185

Zapatero Osorio, M. R., Martín, E. L., \& Rebolo, R. 1997, A\&A, 323, 105 